\title{
IHH Complicated Consumptive Hypothyroidism: A Case Report and Literature Review
}

Qianlong $\mathrm{Liu}^{1}$, Xin $\mathrm{He}^{1}$, $\mathrm{Na} \mathrm{Liu}^{1}$, Jing $\mathrm{Shi}^{1}$, Yan Geng ${ }^{1}$, and Xinkui Guo ${ }^{1}$

${ }^{1}$ Xi'an Jiaotong University Second Affiliated Hospital

February 10, 2021

\begin{abstract}
Through a case of IHH complicated consumptive hypothyroidism and literature review, this study highlight better treatment protocol for these rare patients. It is helpful that simultaneous anti-tumor and thyroid replacement make diffuse IHH patients with consumptive hypothyroidism recovery better.
\end{abstract}

\section{Hosted file}

Manuscript.pdf available at https://authorea.com/users/394832/articles/508185-ihhcomplicated-consumptive-hypothyroidism-a-case-report-and-literature-review

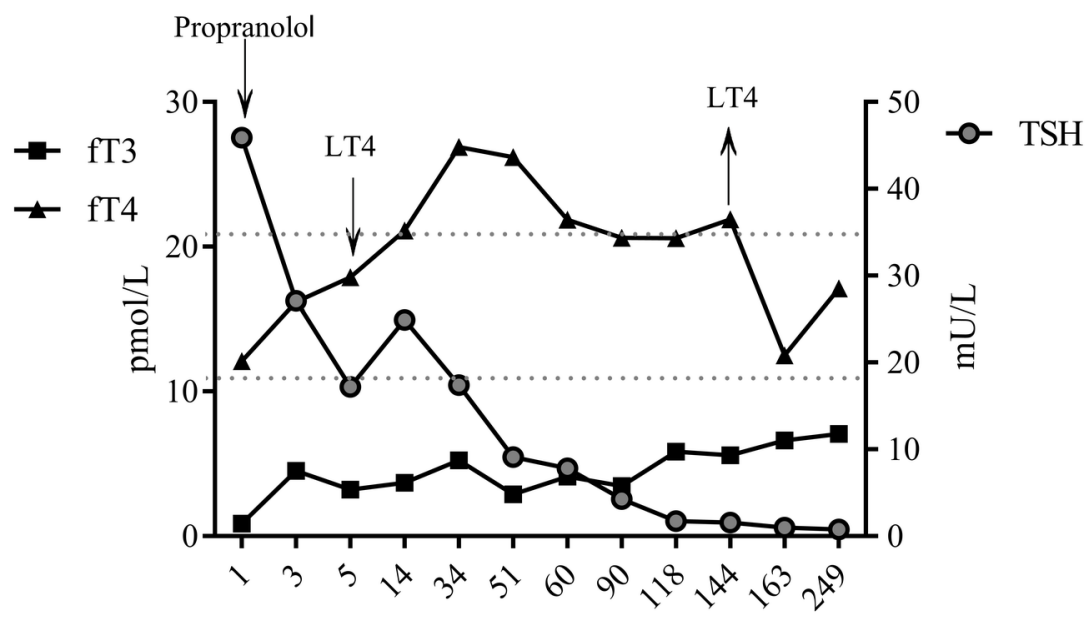

Days after treatment 


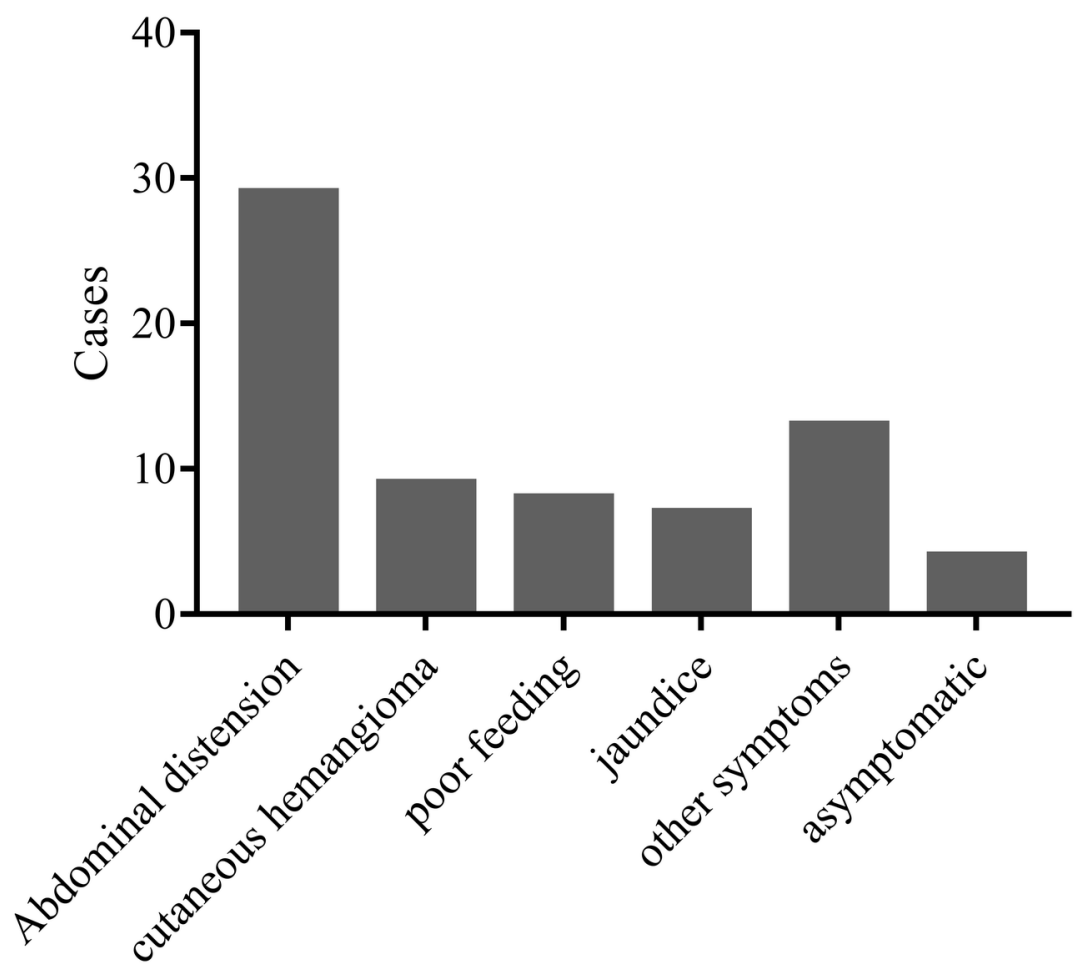

Symptoms 

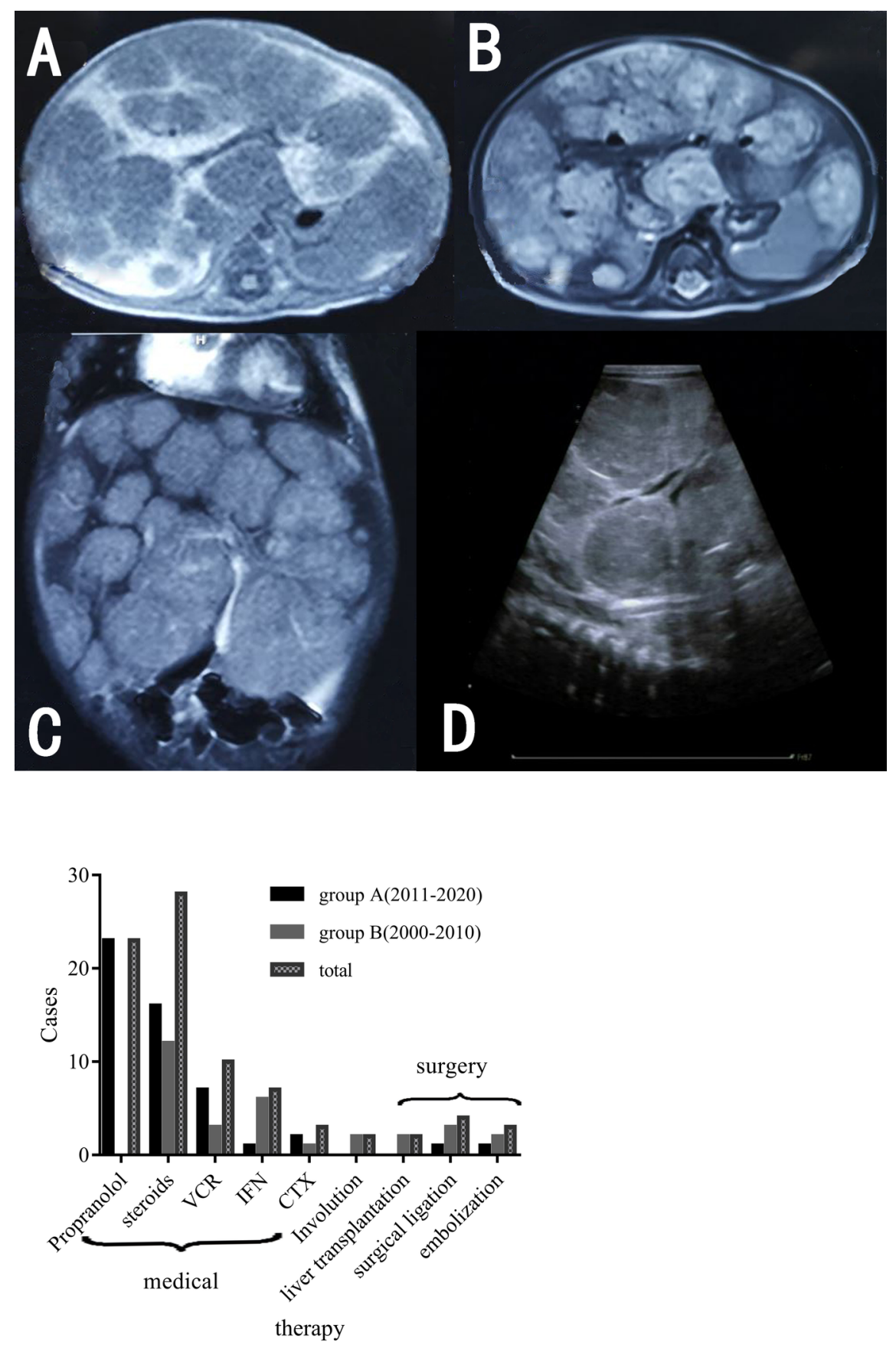